\title{
Recipient Vessel Selection in Immediate Breast Reconstruction with Free Abdominal Tissue Transfer after Nipple-Sparing Mastectomy
}

\author{
Sung Jun Yang ${ }^{1}$, Jin Sup Eom ${ }^{1}$, Taik Jong Lee ${ }^{1}$, Sei Hyun Ahn², Byung Ho Son ${ }^{2}$ \\ Departments of ${ }^{1}$ Plastic Surgery, and ${ }^{2}$ General Surgery, Asan Medical Center, University of Ulsan College of Medicine, Seoul, Korea
}

Background Nipple-sparing mastectomy (NSM) is gaining popularity due to its superior aesthetic results. When reconstructing the breast with free abdominal tissue transfer, we must readdress the recipient vessel, because NSM can cause difficulty in access to the chest vessel.

Methods Between June 2006 and March 2011, a total of 92 women underwent NSM with free abdominal tissue transfer. A lateral oblique incision was used for the nipple-sparing mastectomy. For recipient vessels, the internal mammary vessels were chosen if the mastectomy flap did not block access to the vessels. If it did, the thoracodorsal vessels were used. Age, degree of breast ptosis, weight of the mastectomy specimen, and related complications of the internal mammary vessel group and the thoracodorsal vessel group were compared.

Results Thoracodorsal vessels were used as recipient vessels in 59 cases, and internal mammary vessels in 33 cases including 4 cases with perforators of the internal mammary vessels. Breast reconstruction was successful in all cases except one case involving a total flap failure, which was replaced by a silicone gel implant. The internal mammary group and the thoracodorsal group were similar in terms of age, height, breast weight, and degree of ptosis. The flap related complications such as flap loss and take-back operation rates were not significantly different between the two groups. The rate of nipple necrosis was higher in the internal mammary group. Conclusions The thoracodorsal vessels could produce comparable outcomes in breast reconstruction after nipple-sparing mastectomies. If access to internal mammary vessels is difficult, the thoracodorsal vessel can be a better choice.

Keywords Mammaplasty / Mastectomy / Free tissue flaps
Correspondence: Jin Sup Eom Department of Plastic Surgery, Asan Medical Center, University of Ulsan College of Medicine, 88 Olympic-ro 43-gil, Songpa-gu, Seoul 138-736, Korea

Tel: +82-2-3010-3602

Fax: +82-2-476-7471

E-mail:jinsupp@amc.seoul.kr

\section{INTRODUCTION}

Skin-sparing mastectomy was a breakthrough technique that greatly improved breast reconstruction aesthetic results $[1,2]$ and increased the advantages of immediate reconstruction. It is oncologically safe [3], and now is one of the most widely used mastectomy methods for immediate breast reconstruction. An- other mastectomy option that can preserve the whole native skin envelope is nipple-sparing mastectomy. Although concerns have been raised regarding the oncological safety of this technique [4-12], the aesthetic results seem to be obviously superior [10,13-17]. The nipple-areola complex is much more difficult to reconstruct than the breast mound. The shape and consistency of the breasts reconstructed with autologous tissue are very similar 
to the native breast. However, the shape and color of the reconstructed nipple is not as similar $[18,19]$, and the texture of the reconstructed nipple-areola complex can differ from the existing one as it is usually made using the skin from the abdomen or back. Saving the nipple-areola complex is of a great benefit to patients, so more effort should be made to use nipple-sparing mastectomies.

A nipple-sparing mastectomy (NSM) can provide the best skin envelope for implant-based reconstruction because the whole breast covering remains intact. This is also true in microvascular breast reconstruction using free transverse rectus $a b$ dominis musculocutaneous (TRAM) or deep inferior epigastric artery perforator (DIEP) flaps. The combination of a NSM and free abdominal tissue transfer can provide the optimal skin envelope and breast mound $[13,14]$. Although this combination of procedures is currently uncommon, its popularity is expected to rapidly increase. Applying this combination requires particular considerations in terms of recipient vessels, a monitoring window and managing any nipple-areola complex necrosis. However, few studies have reported on issues surrounding the use of this combined procedure.

The present report describes our experience with breast reconstruction using free abdominal tissue transfer after nipplesparing mastectomy, with a focus on recipient vessel choice and complications.

\section{METHODS}

Between June 2006 and March 2011, a total of 92 women underwent NSMs with immediate breast reconstruction using free abdominal tissue transfer. Cases involving other reconstructive techniques were excluded from the present analysis. The mastectomy and reconstruction was unilateral in all cases. The flaps used for reconstruction were muscle-sparing free TRAM flaps in 38 women, DIEP flaps in 52 women, and superficial inferior epigastric artery (SIEA) flaps in two women.

Every woman was a candidate for a nipple-sparing mastectomy, unless there was evidence of direct involvement of the breast cancer with the nipple-areola complex. The final decision to spare the nipple-areola complex was based on intraoperative pathologic evaluation of breast specimens from the undersurface of the nipple. The NSM incision was a lateral oblique incision commencing from the lateral end of the areola and extending to the anterior axillary line (Fig. 1A). The usual length of the incision was 7 to $9 \mathrm{~cm}$. The incision did not interrupt the border of the areola and did not extend into the medial skin.

Recipient vessels were either the internal mammary vessels or the thoracodorsal vessels, with the former being the first con-

\section{Fig. 1. Operative procedure}

(A) The mastectomy was accomplished using a single incision from the lateral border of the areola to the anterior axillary line. No circumareola or medial extension was made. (B) The mastectomy flap was retracted and the lateral border of the sternum was exposed sufficiently for microsurgery. (C) The deep inferior epigastric artery perforator flap pedicle was anastomosed to the internal mammary vessels using an intercostal approach.
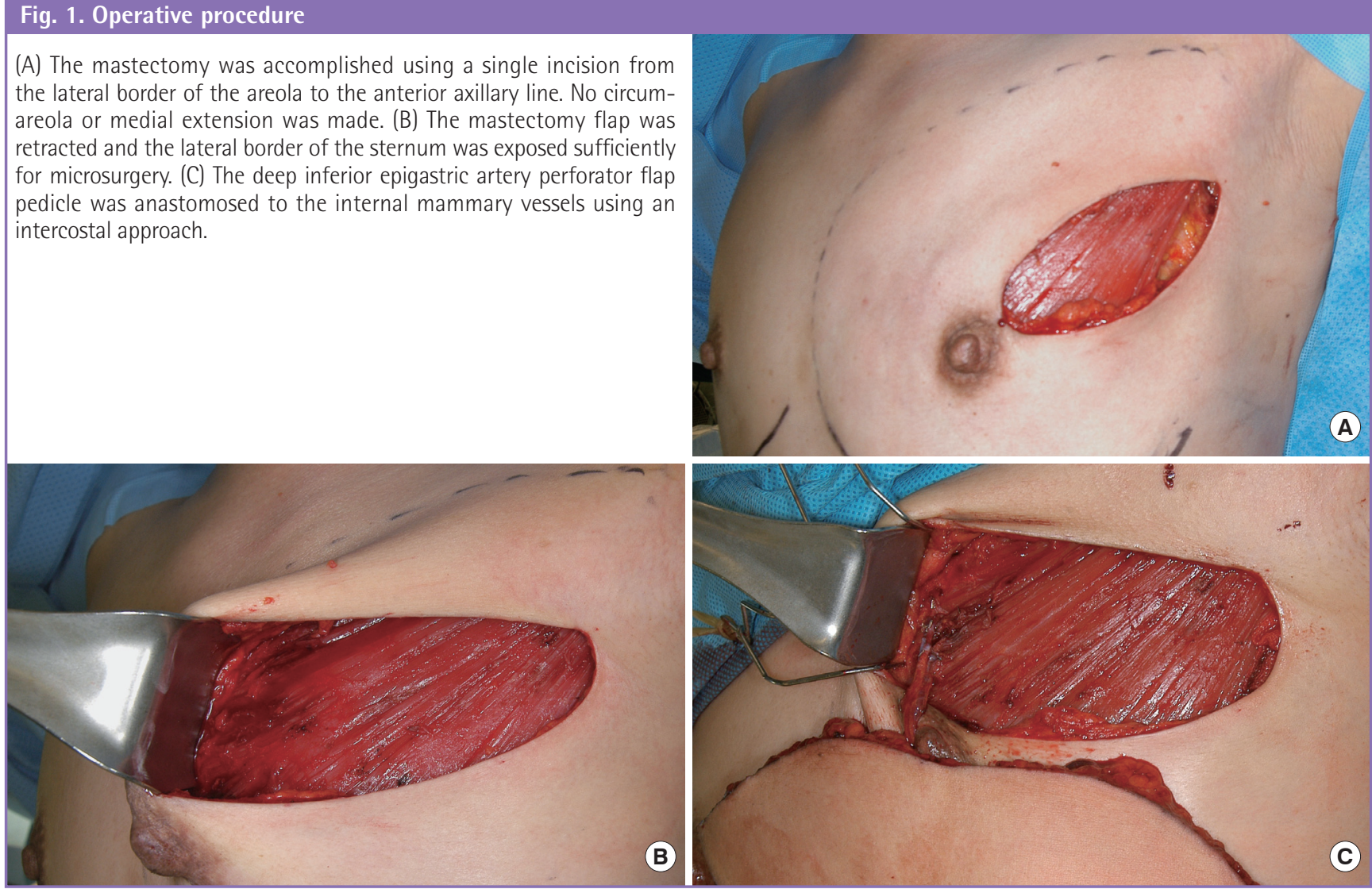
sidered. Retraction of the skin envelope was aimed to expose the lateral sternum border with a perpendicular view (Fig. 1B), and if it was possible, the internal mammary vessels were chosen for the recipient. A perforator artery from the second intercostal space was investigated for use as a recipient vessel, and was deemed large enough to use in microanastomosis if the diameter of the artery exceeded $1 \mathrm{~mm}$ (Fig. 1C). If the diameter was less, the widest intercostal space was divided to approach the internal mammary vessels. A chest X-ray was an excellent indicator of the width of the intercostal spaces, and the second and the third intercostal space was most commonly adopted. Splitting of the pectoralis major muscle and division of the intercostal muscle exposed the internal mammary vessels. If the width between the rib cartilages was greater than $1 \mathrm{~cm}$, microanastomosis was possible. Otherwise, partial resection of the rib cartilage was helpful.
If the lateral border of the sternum was not exposed properly or the skin flap covered the working field, the thoracodorsal vessels were selected as recipient vessels without attempting to lengthen the incision medially (Fig. 2). If the axilla was not exposed sufficiently with retraction of the skin and the pectoralis muscle, the incision was immediately extended laterally to ensure an adequate microanastomosis position. The thoracodorsal vessels were readily identified after axillary lymph node dissection. Without axillary lymph node dissection, exploration of the fatty tissue at the undersurface of the latissimus dorsi muscle was necessary. It was important to obtain maximal vessel length in order to pull the flaps medially to provide sufficient volume in the medial pole. The internal mammary vessels are a better choice for the SIEA flaps since that flap pedicle is shorter than those of the free TRAM or DIEP flap. For the purpose of flap monitoring, a

\section{Fig. 2. Approach for microanastomosis}

(A) The lateral sternum border could be seen under the mastectomy flap, but microanastomosis under the microscope was not possible even with maximal retraction. (B) Access to the axillary vessels was better in this case.
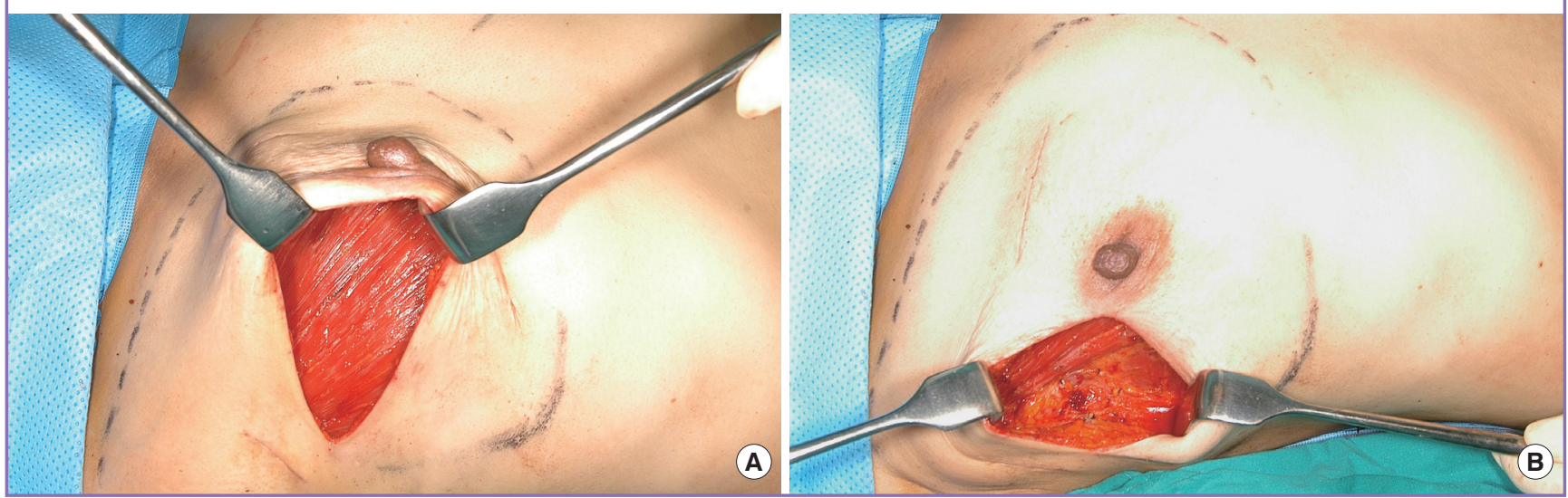

\section{Fig. 3. Preoperative and postoperative photographs}

(A) A 31-year-old woman who underwent a nipple-sparing mastectomy and muscle-sparing free transverse rectus abdominis musculocutaneous (TRAM) flap on the left breast. Although she was nulliparous single, both breasts showed a moderate degree of ptosis. (B) A simultaneous mastopexy of the contralateral breast was performed using a periareolar incision. A periareolar incision was not used on the left breast in order to save the nipple-areola complex, and the skin envelope was redraped upwards over the TRAM flap. The nipple level and degree of ptosis were acceptably symmetrical for the two breasts.
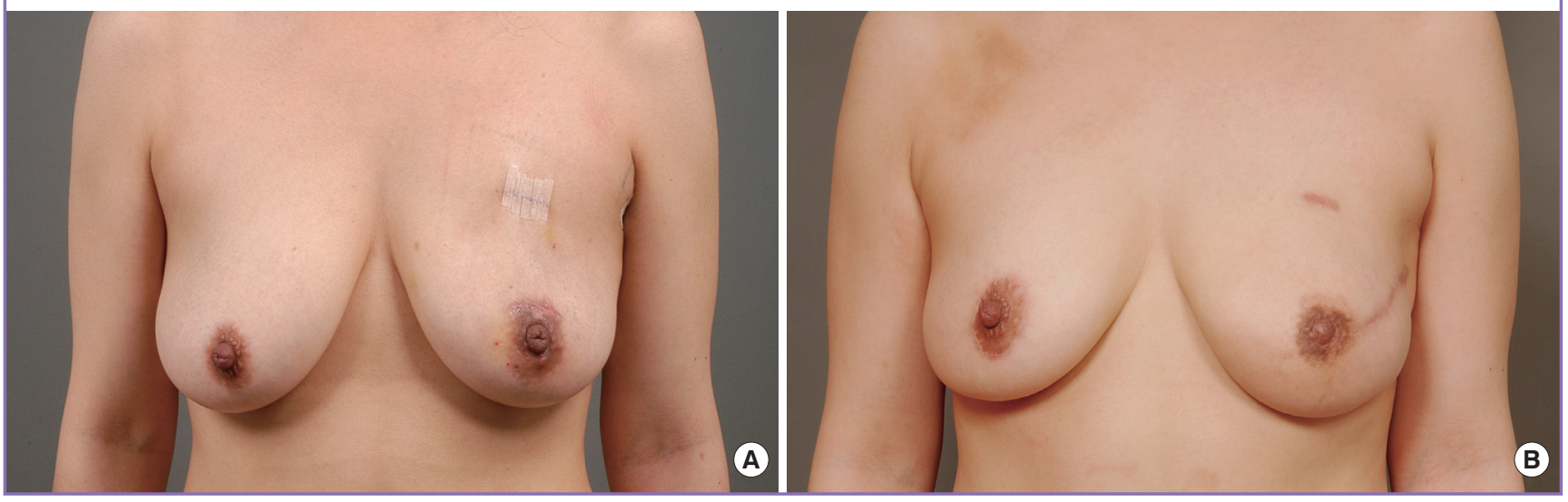
small $(1 \times 2 \mathrm{~cm})$ skin island was preserved and placed between the incision margins. Flap monitoring involved periodic observation of the color and capillary refill of the skin island.

Age, degree of ptosis, weight of the mastectomy specimen, microsurgical outcomes, and complications were compared between the groups for which internal mammary vessels were used and those that had thoracodorsal vessels as recipient vessels. Differences in demographic data between the internal mammary vessel group and thoracodorsal vessel group were evaluated using a $t$ test. Chi-squared tests or Fisher's exact tests were used to determine significant differences in complications such as take back operation, flap failure, nipple necrosis, mastectomy skin necrosis, seroma, and hematoma. Significance was determined by a value of $\mathrm{P}=0.05$. All analysis was performed using SPSS ver. 18.0 (IBM Co., Armonk, NY, USA).

\section{RESULTS}

Breast reconstruction with microsurgical transfer of abdominal tissue was accomplished as planned in all cases (Fig. 3), except one patient from the internal mammary group who experienced a total flap failure that was replaced with a silicone gel implant. Recurrence of breast cancer was not observed in any patient during the follow-up period (5-34 months, mean 18.1 months).

Appropriate exposure of the internal mammary vessels or their perforators was possible in 33 women who were assigned to the internal mammary vessel group, while the axilla was explored in the remaining 59 women who were assigned to the thoracodorsal vessel group. Among the 33 cases of the internal mammary vessel group, the internal mammary perforator was used in 4 cases. The internal mammary and thoracodorsal groups were similar in terms of age, height, breast weight, and degree of ptosis (Table 1).

There were 5 cases of venous congestion which required takeback operations. Reanastomosis of the vein resulted in the flap

Table 1. Comparison of the baseline characteristics between the two groups

\begin{tabular}{|c|c|c|c|}
\hline & $\begin{array}{l}\text { IMA }(n=29) \text { or } \\
\text { IMAp }(n=4)\end{array}$ & $\begin{array}{c}\text { TDA } \\
(n=59)\end{array}$ & P-value \\
\hline Age (yr) & 43.9 & 42.6 & 0.628 \\
\hline Height (cm) & 157.2 & 155.3 & 0.121 \\
\hline Degree of ptosis ${ }^{\text {a) }}$ & 2.50 & 2.62 & 0.108 \\
\hline Body mass index $\left(\mathrm{kg} / \mathrm{cm}^{2}\right)$ & 22.56 & 23.28 & 0.628 \\
\hline $\begin{array}{l}\text { Weight of mastectomy } \\
\text { specimen }(\mathrm{g})\end{array}$ & 343.4 & 439.7 & 0.589 \\
\hline \multicolumn{4}{|c|}{$\begin{array}{l}\text { Continuous variables, T-test. } \\
\text { IMA, internal mammary vessels; IMAp, perforator of IMA; TDA, thoracodorsal vessels. } \\
\text { a) Degree of breast ptosis was graded as } 1,2 \text {, or } 3 \text {, following Baker's ptosis grades } \\
\text { of } A, B \text {, or } C \text {, respectively. }\end{array}$} \\
\hline
\end{tabular}

being salvaged in 4 of those cases, whereas the flap had to be sacrificed in the other case. The rate of take-back surgery was $9.1 \%$ in the internal mammary vessel group and 3.4\% in the thoracodorsal vessel group (Table 2). Statistical analysis using the chisquared method showed that there was no significant difference between the two groups in terms of incidence of flap failure, mastectomy skin necrosis, seroma, or hematoma. In terms of a nipple necrosis, there was a significant difference between the internal mammary group and the thoracodorsal group. In one internal mammary group case, a wide area of the skin envelope including the nipple $(12 \times 7 \mathrm{~cm})$ became necrotic. However, necrosis was anticipated during the operation, and the skin of the flap was not deepithelized and was buried under the skin envelope. Two weeks after surgery, the necrotic skin was debrided and replaced with the buried skin.

In 9 women, simultaneous reduction or mastopexy of the contralateral breast was performed using a vertical mammaplasty design. The vertical design could not be applied to the reconstructed breast because a periareolar incision would lead to excision of the nipple-areola complex. Instead of lifting the areola using a skin excision, the areola was relocated by redraping the mastectomy skin envelope over the flap in an upwards direction to the level of the opposite areola.

\section{DISCUSSION}

Over the last decade, the choice of recipient vessels for breast reconstruction has shifted from the axillary vessels to internal mammary vessels $[20,21]$. The advantages of using the internal mammary vessels include more stable microsurgical results, effectiveness in placing volume in the medial pole of the breast and a simpler surgical procedure. Consequently, thoracodorsal vessels are becoming considered as a second choice. However, with the application of the nipple-sparing mastectomy, more frequent use of thoracodorsal vessels should be considered. In the present series, there was no absolute indicator for choosing

Table 2. Comparison of the two groups' complications

\begin{tabular}{|c|c|c|c|}
\hline & $\begin{array}{l}\text { IMA }(n=29) \text { or } \\
\text { IMAp }(n=4)\end{array}$ & $\begin{array}{c}\text { TDA } \\
(n=59)\end{array}$ & P-value \\
\hline Take-back operation & $3(9.1)$ & $2(3.4)$ & 0.201 \\
\hline Flap failure & 1 & 0 & 0.337 \\
\hline Nipple necrosis & $8(24.2)$ & $4(6.8)$ & 0.042 \\
\hline Mastectomy skin necrosis & $4(12.1)$ & $5(10.1)$ & 0.157 \\
\hline Seroma & $2(6.1)$ & $3(5.1)$ & 0.278 \\
\hline Hematoma & $2(6.1)$ & $3(5.1)$ & 0.278 \\
\hline \multicolumn{4}{|c|}{$\begin{array}{l}\text { Values are presented as number (\%). Statistical analysis using the chi-squared test } \\
\text { showed there were no significant differences between the two groups for each } \\
\text { complication rate. Categorical variables, chi-squared test or Fisher's exact test. } \\
\text { IMA, internal mammary vessels; IMAp, perforator of IMA; TDA, thoracodorsal vessels. }\end{array}$} \\
\hline
\end{tabular}


which vessels would be used as the recipient. The vessels were solely chosen by the surgeon's familiarity with the microvascular surroundings. Some surgeons might be comfortable with the narrow space partially covered with a skin flap and choose the internal mammary vessel more frequently, and some others might not be. Of course, there might be some extreme cases in which the exposure of the sternal margin is almost impossible without additional incision. Our data shows that regardless of the vessel that was chosen, there was no difference in the microsurgical outcomes or flap related complications. The rate of venous congestion and flap failure when using thoracodorsal vessels was in the acceptable range. It is well known that the thoracodorsal vessels are probably among the most reliable recipient vessels in the human body.

Internal mammary vessels could be used more frequently if surgeons could cope with some discomfort during microanastomosis. Extension of the incision to the medial skin may be helpful in approaching the internal mammary vessels. However, the merits of the internal mammary vessels do not seem to outweigh the unnecessary scars interrupting the breast subunit and the increased risk of nipple necrosis. The SIEA flap is an exception since the pedicle is much shorter than the deep inferior epigastric artery, and therefore the recipient vessels should be the internal mammary vessels. A SIEA flap should not be planned if the internal mammary vessels are not available.

We found that a small skin island served as an excellent monitoring window and reflected the exact status of flap vascularity. Although there are many other techniques for monitoring a buried flap, the skin island is the most reliable and economical [22]. There were 5 cases of venous congestion on the day after surgery, and 4 were salvaged with take-back operations. The skin island window was critical in accurate early detection of flap compromise. However, one major drawback of skin island monitoring is inducing flatness after excision. It is difficult to redrape the skin envelope after excision of the skin island because the envelope is already fixed to the flap. Therefore, the skin island width should be less than $1 \mathrm{~cm}$, and it should be located as laterally as possible. A width of $1 \mathrm{~cm}$ is sufficient to monitor flap color and apply leeches if required. However, a skin island that is too small can be a problem since a bruise or blood clot could disguise the true flap color.

Unfortunately, a classical breast reduction incision cannot be used in nipple-sparing mastectomy. A NSM should be abandoned if breast ptosis is severe. However, if ptosis is moderate, the nippleareola complex can be relocated by upward redraping of the mastectomy skin envelope. Ptosis of the reconstructed breast can be controlled by elevation and fixation of the flap to the chest wall, and the nipple-areola complex may be sutured to the flap at the desired level.

The most serious problem associated with NSM is necrosis of the preserved nipple or areola. Loss of the nipple after a NSM will result in a worse outcome than a skin-sparing mastectomy. There is no abdominal skin occupying the areola area and scarred tissue around the nipple would counteract nipple reconstruction. Partial loss of the areola skin will also lead to disfiguring depigmentation. In cases where the surgeon believes the nipple-areola complex may not survive, skin banking under the mastectomy skin envelope is a useful approach $[23,24]$. The nipple and areola are more vulnerable to necrosis than the mastectomy skin flap because they are sitting on the most distal part of the preserved skin. Furthermore, when the internal mammary vessels are used as recipient vessels, the mastectomy skin flap should be retracted with some tension for more than one hour. While it is not clear whether this influences survival of the nipple-areola complex, survival of the nipple and areola is more important than the selection of recipient vessels, as patients expect a benefit following a nipple-sparing mastectomy.

\section{REFERENCES}

1. Carlson GW. Skin sparing mastectomy: anatomic and technical considerations. Am Surg 1996;62:151-5.

2. Carlson GW, Bostwick J 3rd, Styblo TM, et al. Skin-sparing mastectomy. Oncologic and reconstructive considerations. Ann Surg 1997;225:570-5.

3. Park EH, Kim JH, Son BH, et al. Recurrence and survival analysis in skin-sparing mastectomy with immediate reconstruction compared with conventional mastectomy. J Breast Cancer 2005;8:178-85.

4. Wertheim U, Ozzello L. Neoplastic involvement of nipple and skin flap in carcinoma of the breast. Am J Surg Pathol 1980;4:543-9.

5. Andersen JA, Gram JB, Pallesen RM. Involvement of the nipple and areola in breast cancer. Value of clinical findings. Scand J Plast Reconstr Surg 1981;15:39-42.

6. Morimoto T, Komaki K, Inui K, et al. Involvement of nipple and areola in early breast cancer. Cancer 1985;55:2459-63.

7. Laronga C, Kemp B, Johnston D, et al. The incidence of occult nipple-areola complex involvement in breast cancer patients receiving a skin-sparing mastectomy. Ann Surg Oncol 1999;6:609-13.

8. Cense HA, Rutgers EJ, Lopes Cardozo M, et al. Nipple-sparing mastectomy in breast cancer: a viable option? Eur J Surg Oncol 2001;27:521-6.

9. Gerber B, Krause A, Reimer T, et al. Skin-sparing mastectomy with conservation of the nipple-areola complex and au- 
tologous reconstruction is an oncologically safe procedure. Ann Surg 2003;238:120-7.

10. Mosahebi A, Ramakrishnan V, Gittos M, et al. Envelope mastectomy and immediate reconstruction (EMIR), improving outcome without oncological compromise. J Plast Reconstr Aesthet Surg 2006;59:1025-30.

11. Petit JY, Veronesi U, Rey P, et al. Nipple-sparing mastectomy: risk of nipple-areolar recurrences in a series of 579 cases. Breast Cancer Res Treat 2009;114:97-101.

12. Gerber B, Krause A, Dieterich M, et al. The oncological safety of skin sparing mastectomy with conservation of the nippleareola complex and autologous reconstruction: an extended follow-up study. Ann Surg 2009;249:461-8.

13. Nahabedian MY, Tsangaris TN. Breast reconstruction following subcutaneous mastectomy for cancer: a critical appraisal of the nipple-areola complex. Plast Reconstr Surg 2006; 117:1083-90.

14. Mosahebi A, Ramakrishnan V, Gittos M, et al. Aesthetic outcome of different techniques of reconstruction following nipple-areola-preserving envelope mastectomy with immediate reconstruction. Plast Reconstr Surg 2007;119:796-803.

15. Denewer A, Farouk O. Can nipple-sparing mastectomy and immediate breast reconstruction with modified extended latissimus dorsi muscular flap improve the cosmetic and functional outcome among patients with breast carcinoma? World J Surg 2007;31:1169-77.

16. Dao TN, Verheyden CN. TRAM flaps: a reconstructive op- tion after bilateral nipple-sparing total mastectomy. Plast Reconstr Surg 2005;116:986-92.

17. Suh HS, Lee TJ, Eom JS, et al. Immediate breast reconstruction with tram flap after nipple-areolar sparing mastectomy. J Korean Soc Plast Reconstr Surg 2006;33:570-6.

18. Jabor MA, Shayani P, Collins DR Jr, et al. Nipple-areola reconstruction: satisfaction and clinical determinants. Plast Reconstr Surg 2002;110:457-63.

19. Shestak KC, Gabriel A, Landecker A, et al. Assessment of long-term nipple projection: a comparison of three techniques. Plast Reconstr Surg 2002;110:780-6.

20. Kronowitz SJ, Kuerer HM, Hunt KK, et al. Impact of sentinel lymph node biopsy on the evolution of breast reconstruction. Plast Reconstr Surg 2006;118:1089-99.

21. Moran SL, Nava G, Behnam AB, et al. An outcome analysis comparing the thoracodorsal and internal mammary vessels as recipient sites for microvascular breast reconstruction: a prospective study of 100 patients. Plast Reconstr Surg 2003;111: 1876-82.

22. Ferguson RE Jr, Yu P. Techniques of monitoring buried fasciocutaneous free flaps. Plast Reconstr Surg 2009;123:525-32.

23. Kovach SJ, Georgiade GS. The "banked" TRAM: a method to insure mastectomy skin-flap survival. Ann Plast Surg 2006; 57:366-9.

24. Liao EC, Labow BI, May JW Jr. Skin banking closure technique in immediate autologous breast reconstruction. Plast Reconstr Surg 2007;120:1133-6. 